\title{
Análise da velocidade linear em jogadores de futebol a partir de dois métodos de avaliação
}

\author{
Analysis of the linear sprint speed of soccer players using \\ two assessment methods
}

\author{
Bruno Natale Pasquarelli 1,2 \\ Luiz Cláudio Reeberg Stanganelli 1,2 \\ Antonio Carlos Dourado 1,2 \\ Mathias Roberto Loch ${ }^{1}$ \\ César Augusto de Andrade ${ }^{2,3}$
}

\begin{abstract}
1 Universidade Estadual de Londrina, Londrina, Paraná, Brasil.
\end{abstract}

2 Centro de Excelência Esportiva (Ministério do Esporte / Secretaria Nacional de Alto Rendimento)

3 Faculdade de Motricidade Humana, Lisboa, Portugal.

Recebido em 29/05/08 Revisado em 13/08/08 Aprovado em 14/04/09
Resumo - O presente estudo teve como objetivo analisar o desempenho de jogadores de futebol no teste de velocidade de $40 \mathrm{~m}$ a partir de dois métodos de avaliação, de acordo com o desempenho no teste e posição no jogo. Foram analisados 154 atletas profissionais de futebol da $1^{\text {a }}$ Divisão do Estado do Paraná, entre os anos de 2002 a 2006. Os atletas realizaram o teste de velocidade linear de 40 metros, usando o sistema fotoelétrico para medidas de velocidade posicionados na linha de início $(0 \mathrm{~m})$ e nos 10, 20, 30 e 40m. Os atletas, divididos em subgrupos de acordo com o desempenho (tempo final) no teste de velocidade de 40m, foram classificados em: Mais Velozes (tempo entre 4,877 - 5,174s); Intermediários (tempo entre 5,175 - 5,474s); e Menos Velozes (tempo entre 5,475 - 5,766s). Os mesmos 154 atletas, divididos por posição, foram classificados como: Zagueiros, Laterais, Volantes, Meias e Atacantes. Nos dois métodos, foi utilizada ANOVA (one-way) para comparar o tempo médio entre os subgrupos nas diferentes distâncias. No método que separa os atletas pelo desempenho, o teste de Tukey indicou que todos os subgrupos foram diferentes entre si. Para os subgrupos divididos por suas posições no jogo, apesar de serem identificadas visualmente, não foram encontradas diferenças estatísticas. Assim sendo, pode-se inferir que, com base nesses dois métodos de análise da velocidade linear de jogadores de futebol, é possível identificar aspectos relevantes do processo da prescrição do treinamento de velocidade para cada subgrupo e de acordo com suas funções.

Palavras-chave: Futebol; Avaliação; Esportes.

Abstract - The aim of this study was to analyze the performance of soccer players in a 40-m sprint test using two different assessment methods. A total of 154 professional soccer players from the 1st Division of Paraná State were studied between 2002 and 2006. Sprint speed was measured with a set of photocells positioned on the start line $(0 \mathrm{~m})$ and at 10,20, 30 and 40 $m$. The athletes were divided into three subgroups according to performance (final time) in the 40-m sprint test and were classified as fast (4.877-5.17 s), intermediate fast (5.175-5.474 s), and slow (5.475-5.766 s). The same 154 athletes were divided according to their field positions into center back, back side, defensive midfielder, offensive midfielder, and forward. In both methods, ANOVA (one-way) was used for the comparison of average time between subgroups at the different distances. For the method classifying athletes according to performance, the Tukey post-hoc test showed a significant difference between all subgroups. Differences, although not significant, were observed between subgroups classified according to field position during the match. In conclusion, the two methods for the analysis of linear sprint speed in soccer players permit the identification of relevant aspects that could be applied to the prescription of training velocity for each subgroup according to their field position during the match.

Key words: Soccer; Evaluation; Sports. 


\section{INTRODUÇÃO}

A distância percorrida por um jogador de futebol de alto nível em uma partida varia entre $10-12 \mathrm{~km}^{1-5}$. Os sprints contribuem, em média, entre $1-11 \%$ da distância total percorrida em uma partida ${ }^{3,6-8}$, equivalente a $0,11-0,7 \mathrm{~km}^{2,3,5,6}$. No entanto, são requeridos nos momentos mais cruciais do jogo, podendo influenciar diretamente na recuperação da posse de bola na defesa e nas jogadas decisivas no ataque; portanto, podem ser importantes para decidir o resultado de uma partida ${ }^{6,9-12}$.

Um sprint ocorre, aproximadamente, a cada $90 s^{6,13}$ e cada jogador realiza cerca de 10 - 40 sprints de alta velocidade por partida ${ }^{4,14}$, com tempo médio de duração de $2 s^{14}$. Durante uma partida, na maioria das vezes, os jogadores realizam sprints menores que $30 \mathrm{~m}^{14,15}$. De acordo com o estudo de Valquer et al. ${ }^{15}, 96 \%$ dos sprints são menores que $30 \mathrm{~m}$, sendo $48 \%$ destes, menores que $10 \mathrm{~m}$. Desse modo, alguns autores ressaltam que a fase inicial de um sprint é uma variável importante no futebol moderno ${ }^{1,4,16,17}$. Stølen et al. ${ }^{1}$ acrescentam que os jogadores mais velozes chegam, em média, $1 \mathrm{~m}$ à frente dos jogadores menos velozes em uma distância curta de apenas $10 \mathrm{~m}$, o que pode ser importante nas situações de duelos, influenciando o resultado da partida.

A verificação dessa capacidade permite avaliar o estado atual em que o atleta se encontra nessa variável, estruturar as sessões de treinamento, baseandose nos resultados dos testes e examinar se os estímulos do treinamento foram suficientes para causar uma adaptação positiva no atleta ${ }^{18}$. Caracterizando a influência da velocidade nos esportes coletivos com características intermitentes, incluindo o futebol, torna-se importante a verificação desta capacidade física em jogadores dessa modalidade.

Dois métodos são mais comumente utilizados para avaliação dos resultados no teste de velocidade. O primeiro utiliza restritamente o tempo final como ferramenta analítica do desempenho do atleta, no teste de velocidade, em uma determinada distância. No entanto, esse método não permite identificar pontos fracos que influenciaram o desempenho durante o teste, sendo que, dois atletas podem apresentar tempos similares no teste de velocidade linear de 40m e uma diferença significativa na aceleração nos $10 \mathrm{~m}$ iniciais. Portanto, esse método pode ser limitado quando usado para direcionar o treinamento para melhora desta única variável (tempo nos $40 \mathrm{~m})^{18}$.

O segundo e mais recente método de avaliação da velocidade linear, proposto por Brown et al. ${ }^{18}$, separa os atletas em subgrupos estabelecidos pelo tempo final no teste, independente de suas posições, o que pode ser importante para determinar o foco do treinamento de acordo com a necessidade de cada subgrupo. E também, avalia o desempenho dos atletas em diferentes distâncias $(0-10 \mathrm{~m}, 10-20 \mathrm{~m}$, $20-30 \mathrm{~m}, 30-40 \mathrm{~m}$ ), permitindo que o tempo seja analisado cumulativamente (somando o tempo de cada parcial) e também a cada $10 \mathrm{~m}$ separadamente. De acordo com os mesmos autores, este método permite verificar com mais sensibilidade os pontos fracos que influenciam o resultado do teste, quais os efeitos advindos do treinamento aplicado e também pode direcionar o treinamento de acordo com a necessidade de cada atleta ou subgrupo.

Um estímulo de treinamento específico de velocidade é importante para melhora dessa capacidade ${ }^{18}$. Esses estímulos podem ser direcionados à melhora de múltiplas variáveis que afetam o desempenho na velocidade, sendo elas de caráter fisiológico/condicionante ou biomecânico/coordenativo ${ }^{12,19}$. Di Salvo $\&$ Pigozzi ${ }^{20}$ afirmam que, no futebol, o treinamento individualizado por posições, de acordo com as diferentes demandas bioenergéticas requeridas no jogo, é um método que pode otimizar o desempenho de todos os jogadores da equipe. Dessa maneira, contrasta com o método proposto por Brown et al. ${ }^{18}$ para avaliação da velocidade, pois este método separa os subgrupos pelo desempenho no teste independente das funções que cada jogador exerce.

A utilização da avaliação física é importante no planejamento de curto, médio e longo prazo no futebol e nos demais esportes. No entanto, é necessária a utilização de métodos que analisem e utilizem os resultados obtidos nos testes para tal fim. Até o momento, não foram encontrados dados na literatura que utilizem o método de Brown et al. ${ }^{18}$ para analisar a velocidade linear de jogadores de futebol, dividindo os subgrupos pelo tempo final, como proposto pelo autor, e por suas posições no jogo. Sendo assim, torna-se importante a análise do desempenho de jogadores de futebol no teste de velocidade linear a partir destes dois métodos.

Portanto, o objetivo do presente estudo foi o de analisar o desempenho de jogadores de futebol no teste de velocidade de $40 \mathrm{~m}$, utilizando o método de Brown et al. ${ }^{18}$, bem como de acordo com suas posições específicas no jogo.

\section{PROCEDIMENTOS METODOLÓGICOS}

Este estudo de caráter descritivo analítico analisou a velocidade linear de jogadores de futebol através do teste de $40 \mathrm{~m}$, utilizando dois métodos de avaliação. 


\section{Amostra}

A amostra foi composta de 154 atletas profissionais de futebol da $1^{a}$ Divisão do Estado do Paraná, entre os anos de 2002 a 2006. A Tabela 1 mostra algumas informações antropométricas da amostra investigada. Para os indivíduos que se repetiam ao longo dos anos ou da temporada, foi considerado apenas o último teste realizado no Centro de Excelência Esportiva (CENESP); desse modo, não foram analisados os dados de um mesmo atleta mais de uma vez. O presente estudo foi aprovado pelo Comitê de Ética em Pesquisa com Seres Humanos da Universidade Estadual de Londrina, sob o protocolo número 062/01.

Tabela 1. Características antropométricas dos jogadores de futebol (Média \pm DP).

\begin{tabular}{lcc}
\hline Características & $\mathrm{n}$ & Média \pm DP \\
\hline Idade (anos) & 151 & $23,9 \pm 3,9$ \\
Estatura $(\mathrm{cm})$ & 152 & $177,3 \pm 6,2$ \\
Massa Corporal $(\mathrm{kg})$ & 152 & $73,3 \pm 6,6$ \\
SM7DC $(\mathrm{mm}) *$ & 132 & $63,6 \pm 18,2$ \\
\hline
\end{tabular}

*Somatória das dobras cutâneas: tríceps, subescapular, bíceps, suprailíaca, abdominal, coxa e panturrilha medial.

\section{Procedimentos de Análise}

Todos os testes foram realizados no período vespertino, entre 15 e $18 \mathrm{~h}$. Foram realizados sprints lineares máximos de $40 \mathrm{~m}$, usando o sistema fotoelétrico (marca Greika, modelo WT 3131) para medidas de velocidade posicionados na linha de início $(0 \mathrm{~m})$ e nos 10, 20, 30 e 40m, com altura aproximada de $0,5 \mathrm{~m}$.

Os sujeitos partiram da posição estática, com um dos pés, selecionado pelo próprio atleta, colocado à frente, próximo da linha de início. Para eliminar a influência do tempo de reação nos resultados, os atletas foram orientados a começar o teste quando quisessem e foram instruídos a correr em máxima velocidade, até a passagem pelo último par de fotocélulas. $O$ tempo foi iniciado a partir do primeiro movimento do atleta ao atravessar o primeiro par de fotocélulas posicionado na linha de início. Os atletas realizaram dois sprints máximos com tempo de descanso de, aproximadamente, 10 minutos entre eles. Para análise deste estudo, utilizou-se o melhor resultado no teste realizado.

Para a classificação dos subgrupos, foram utilizados os tempos cumulativos no teste de velocidade de $40 \mathrm{~m}(0-40 \mathrm{~m})$. A diferença entre o valor máximo (5,766s) e mínimo (4,877s) foi de 0,889s. Este valor de 0,889 s foi dividido em três terços, que deu origem a três subgrupos com variação de 0,296s entre eles. Os subgrupos foram classificados como: mais velozes (MaV) ( $n=36)$, com tempo entre 4,877 - 5,174s; intermediário (IM) ( $\mathrm{n}=96)$, com tempo entre 5,175 - 5,474s; e menos velozes $(\mathrm{MeV})(\mathrm{n}=22)$, e tempo entre 5,475 - 5,766s.

Os mesmos jogadores foram divididos em subgrupos de acordo com suas respectivas posições: zagueiros $(n=31)$, laterais $(n=27)$, volantes $(n=28)$, meias $(n=38)$ e atacantes $(n=30)$. Não foram incluídos os goleiros, visto que estes possuem especificidades, tanto no jogo quanto nos treinamentos e que, por essa razão, realizam um trabalho de velocidade diferenciado dos demais jogadores.

Os subgrupos foram criados também para verificar se as diferenças no tempo cumulativo dos $40 \mathrm{~m}$ foram causadas por alguma parcial em particular.

\section{Procedimentos Estatísticos}

Os procedimentos estatísticos foram realizados, usando o software SPSS 12.0 for Windows (SPSS Inc.). Para os subgrupos divididos por meio do desempenho no teste de velocidade linear de 40m, ANOVA (one-way) foi usada para comparar o tempo cumulativo entre os subgrupos e também para comparar os tempos da $1^{\mathrm{a}}, 2^{\mathrm{a}}, 3^{\mathrm{a}}$ e $4^{\mathrm{a}}$ parcial de $10 \mathrm{~m}$ de cada subgrupo. Foi utilizado o teste de comparações múltiplas post-hoc para identificar as possíveis diferenças entre os subgrupos, mediante a aplicação do teste de Tukey. Os mesmos procedimentos estatísticos foram aplicados aos subgrupos divididos de acordo com suas respectivas posições no jogo. O software Epicalc (Epicalc, 2004) foi utilizado para comparar os tempos médios obtidos no presente estudo no teste de velocidade, com outros estudos encontrados na literatura, em diferentes distâncias. Para todos os procedimentos estatísticos, foi adotado um nível de significância de p<0,05.

\section{RESULTADOS}

Os dados descritivos para o tempo médio (parcial e cumulativo) e velocidade a cada $10 \mathrm{~m}$ estão representados na Tabela 2.

Tabela 2. Resultados de tempo e velocidade dos 154 atletas (Média \pm DP).

\begin{tabular}{lcccc}
\hline Variáveis & $10 \mathrm{~m}$ & $20 \mathrm{~m}$ & $30 \mathrm{~m}$ & $40 \mathrm{~m}$ \\
\hline $\begin{array}{l}\text { Tempo } \\
\text { Parcial (s) }\end{array}$ & $1,76 \pm 0,10$ & $1,25 \pm 0,05$ & $1,16 \pm 0,04$ & $1,15 \pm 0,05$ \\
$\begin{array}{l}\text { Tempo } \\
\text { Cumulati- }\end{array}$ & $1,76 \pm 0,10$ & $3,01 \pm 0,13$ & $4,17 \pm 0,15$ & $5,32 \pm 0,18$ \\
$\begin{array}{l}\text { vo (s) } \\
\text { Velocidade } \\
(\mathrm{m} / \mathrm{s})\end{array}$ & $5,70 \pm 0,30$ & $8,04 \pm 0,29$ & $8,62 \pm 0,33$ & $8,73 \pm 0,40$ \\
\hline
\end{tabular}


$\mathrm{Na}$ Tabela 3, estão apresentados os tempos médios de cada parcial para os três subgrupos divididos pelo desempenho ( $\mathrm{MaV}, \mathrm{IM}, \mathrm{MeV})$. Foram encontradas diferenças entre os três subgrupos para cada parcial. O teste post-hoc de Tukey indicou que todos os subgrupos foram significantemente diferentes entre si, em todas as parciais de $10 \mathrm{~m}$.

A Tabela 4 mostra os tempos médios de cada parcial nos subgrupos divididos pelas posições específicas. Não foram encontradas diferenças significativas entre os subgrupos em nenhuma das parciais de $10 \mathrm{~m}$.

Na Tabela 5, estão apresentados os valores de tempo de vários estudos recentes, encontrados na literatura, em diferentes distâncias (10, 20, 30 e $40 \mathrm{~m}$ ) e de diferentes níveis competitivos.
Tabela 3. Parcial de tempo (Média \pm DP) em diferentes distâncias para os subgrupos mais velozes (MaV), intermediário (IM) e menos velozes (MeV).

\begin{tabular}{cccc}
\hline Distância & \multicolumn{3}{c}{ Subgrupos } \\
& MaV & IM & MeV \\
\hline $0-10^{*}$ & $1,66 \pm 0,05$ & $1,76 \pm 0,07$ & $1,88 \pm 0,09$ \\
$10-20^{*}$ & $1,20 \pm 0,03$ & $1,25 \pm 0,03$ & $1,30 \pm 0,05$ \\
$20-30^{*}$ & $1,12 \pm 0,03$ & $1,16 \pm 0,03$ & $1,22 \pm 0,04$ \\
$30-40^{*}$ & $1,10 \pm 0,03$ & $1,15 \pm 0,05$ & $1,20 \pm 0,05$ \\
$0-40^{*}$ & $5,09 \pm 0,06$ & $5,33 \pm 0,09$ & $5,60 \pm 0,09$ \\
\hline
\end{tabular}

$* p<0,05$

Tabela 4. Parcial de tempo (Média \pm DP) em diferentes distâncias para os subgrupos Zagueiros, Laterais, Volantes, Meias e Atacantes.

\begin{tabular}{lccccc}
\hline Distância & \multicolumn{5}{c}{ Posições } \\
\hline $0-10$ & Zagueiros & Laterais & Volantes & Meias & Atacantes \\
$10-20$ & $1,78 \pm 0,11$ & $1,73 \pm 0,09$ & $1,78 \pm 0,09$ & $1,76 \pm 0,10$ & $1,74 \pm 0,09$ \\
$20-30$ & $1,26 \pm 0,04$ & $1,22 \pm 0,04$ & $1,25 \pm 0,04$ & $1,25 \pm 0,05$ & $1,23 \pm 0,04$ \\
$30-40$ & $1,16 \pm 0,04$ & $1,15 \pm 0,04$ & $1,15 \pm 0,05$ & $1,17 \pm 0,04$ & $1,16 \pm 0,05$ \\
$0-40$ & $1,15 \pm 0,05$ & $1,15 \pm 0,06$ & $1,14 \pm 0,06$ & $1,16 \pm 0,05$ & $1,15 \pm 0,05$ \\
\hline
\end{tabular}

Tabela 5. Valores de tempo (Média \pm DP) em diferentes distâncias de outros estudos encontrados na literatura.

\begin{tabular}{|c|c|c|c|c|}
\hline Estudos & Distância (m) & Tempo (s) & $\mathrm{n}$ & Nível competitivo \\
\hline Dourado et al. ${ }^{22}$ & 10 & $1,71 \pm 0,14^{*}$ & 167 & $1^{\underline{a}}$ div. Estad. Junior Brasil \\
\hline Dourado et al. ${ }^{22}$ & 10 & $1,74 \pm 0,11^{*}$ & 230 & $1^{\text {a }}$ div. Estadual Brasil \\
\hline Little \& Williams ${ }^{10}$ & 10 & $1,83 \pm 0,08^{*}$ & 106 & $1^{\mathrm{a}}$ e $2^{\underline{a}}$ div. Inglaterra \\
\hline Newman et al. ${ }^{24}$ & 10 & $1,81 \pm 0,08$ & 14 & Amad. e Universit. \\
\hline Strudwick et al. ${ }^{25}$ & 10 & $1,75 \pm 0,08$ & 19 & 1a div. Inglaterra \\
\hline Wisloff et al. ${ }^{16}$ & 10 & $1,82 \pm 0,30$ & 17 & $1^{\underline{a}}$ div. Noruega \\
\hline Dauty et al. ${ }^{21}$ & 20 & $3,01 \pm 0,10$ & 20 & 1a div. França \\
\hline Wisloff et al. ${ }^{16}$ & 20 & $3,00 \pm 0,30$ & 17 & $1^{\underline{a}}$ div. Noruega \\
\hline Neves et al. ${ }^{23}$ & 30 & $4,16 \pm 0,03$ & 16 & $1^{\mathrm{a}}$ div. Portugal \\
\hline Neto et al. ${ }^{28}$ & 30 & $4,10 \pm 0,10 \#$ & 35 & $1^{\underline{a}}$ div. Junior Brasil \\
\hline Strudwick et al. ${ }^{25}$ & 30 & $4,28 \pm 0,12^{*}$ & 19 & 1a div. Inglaterra \\
\hline Wisloff et al. ${ }^{16}$ & 30 & $4,00 \pm 0,20^{*}$ & 17 & $1^{a}$ div. Noruega \\
\hline Dourado et al. ${ }^{22}$ & 40 & $5,31 \pm 0,26$ & 167 & $1^{a}$ div. Estad. Junior Brasil \\
\hline Dourado et al. ${ }^{22}$ & 40 & $5,31 \pm 0,20$ & 230 & $1^{\mathrm{a}}$ div. Estadual Brasil \\
\hline Reilly et al. ${ }^{6}$ & 40 & $5,51 \pm 0,08^{*}$ & 66 & $1^{\underline{a}}$ div. Inglaterra \\
\hline
\end{tabular}

Valores de tempo do presente estudo: $10 \mathrm{~m}(1,76 \pm 0,10), 20 \mathrm{~m}(3,01 \pm 0,13), 30 \mathrm{~m}(4,16 \pm 0,15), 40 \mathrm{~m}(5,32 \pm 0,18)$.

* $\mathrm{p}<0,01 ; \# \mathrm{p}<0,05$ (comparado com dados deste estudo). 


\section{DISCUSSÃO}

Este estudo de caráter descritivo analítico teve como objetivo analisar a velocidade linear de jogadores de futebol por meio de dois métodos de avaliação.

Quando comparado os resultados médios no teste de velocidade em diferentes distâncias com outros estudos recentes, encontrados na literatura, verificou-se que para a distância de $0-10 \mathrm{~m}$, quatro estudos foram estatisticamente diferentes e quatro não apresentaram diferenças estatísticas. Para a distância de 0-20m, três estudos foram significantemente diferentes do atual e dois não apresentaram diferença estatística significante. Na distância de 0-30m, três estudos apresentaram ser diferentes estatisticamente, sendo que somente um não apresentou diferença significante. E para a distância de $0-40 \mathrm{~m}$, um estudo apresentou diferença estatisticamente significante e dois não apresentaram nenhuma diferença estatística.

Entretanto, deve-se levar em consideração que algumas variáveis possam ter influenciado o desempenho dos atletas, dificultando a comparação entre o presente estudo e a literatura, como por exemplo: número da amostra, nível competitivo e de treinamento, idade dos atletas, tempo de treino, predisposição genética, piso em que foi realizado o teste e o calçado utilizado pelos atletas.

Apesar de a distância no teste de velocidade linear variar em muitos estudos, a distância de $10 \mathrm{~m}$ foi a que mais se repetiu entre os estudos encontrados, visto que é considerada uma importante variável no futebol moderno, e pode ser incluída nos programas de avaliação desta modalidade. Em um estudo recente, utilizando a mesma amostra deste, Pasquarelli et al..$^{29}$ mostraram que os atletas do subgrupo MaV chegaram, em média, 0,75 e 1,59m à frente dos atletas dos subgrupos $\mathrm{IM} \mathrm{e} \mathrm{MeV}$, respectivamente, na distância de 0 a $10 \mathrm{~m}$. No presente estudo, verificou-se, também, uma forte correlação entre os $10 \mathrm{~m}$ correspondentes à aceleração inicial e o tempo obtido ao final dos $30 \mathrm{~m}\left(\mathrm{r}=0,88 ; \mathrm{r}^{2}=0,77\right)$ e $40 \mathrm{~m}\left(\mathrm{r}=0,77 ; \mathrm{r}^{2}=0,60\right)$.

Houve uma diferença significante entre os três subgrupos classificados pelo desempenho no teste de velocidade. A Tabela 3 mostra que, o subgrupo $\mathrm{MaV}$ apresentou parciais de tempos menores que os outros dois em todas as distâncias, seguido do subgrupo IM, que apresentou o mesmo resultado em relação ao subgrupo $\mathrm{MeV}$. Isso indica que os atletas mais velozes nos $40 \mathrm{~m}$ são também os mais velozes em todas as distâncias parciais de 10m. Esse resultado corrobora o estudo de Brown et al. ${ }^{18}$.
Em contrapartida, não houve diferenças significantes para os subgrupos por posição em nenhuma das parciais de tempo de $10 \mathrm{~m}$. Porém, a Tabela 4 mostra que os zagueiros parecem ser mais lentos nos 10 e $20 \mathrm{~m}$ iniciais se comparados com os atletas nas outras posições, mas possuem tempos semelhantes nos 30 e 40m. Ao contrário dos laterais, que são mais velozes que os jogadores de outras posições, nos 10 e $20 \mathrm{~m}$ iniciais, mas não mantêm nos 30 e 40m, obtendo resultados parecidos às demais posições. Os volantes, por sua vez, possuem uma fraca aceleração nos $10 \mathrm{~m}$ iniciais, continuam acelerando nos $20 \mathrm{e}$ $30 \mathrm{~m}$, sendo mais velozes nos últimos $10 \mathrm{~m}$, de 30 a $40 \mathrm{~m}$. Os meias possuem um desempenho abaixo da média das outras posições em todos os trechos, sendo os menos velozes nos 30 e $40 \mathrm{~m}$. Os atacantes possuem uma maior capacidade de aceleração nos 10 e $20 \mathrm{~m}$ iniciais e tempos semelhantes nos 30 e $40 \mathrm{~m}$ quando comparado às outras posições. Tal análise foi capaz de mostrar que mesmo não sendo encontradas diferenças estatisticamente significantes entre futebolistas de diferentes posições, é possível identificar pontos fracos e fortes de cada uma delas. Portanto, é conveniente que os treinadores e preparadores físicos compreendam essas diferenças e utilizem os testes de velocidade como ferramenta no planejamento do treinamento de acordo com as características de cada posição, para otimizar o desempenho de todos os atletas de sua equipe.

Pode-se notar que a análise dos dados por esses dois métodos utilizados pode fornecer informações distintas e relevantes. Diferentemente do método por desempenho, o método que separa os subgrupos por posições mostrou que os atletas mais rápidos nas primeiras parciais não são, obrigatoriamente, mais rápidos nas parciais seguintes. Todavia, permite conhecer as características de cada posição no sprint linear de $40 \mathrm{~m}$, sabendo quais os pontos fracos e fortes em cada uma delas. Entretanto, o método que separa os subgrupos pelo desempenho parece estabelecer melhor a diferença entre os atletas, dando mais subsídios para a estruturação do treinamento de acordo com a necessidade de cada um deles.

A utilização dos dois métodos mencionados neste estudo pode auxiliar, durante toda a temporada, treinadores e preparadores físicos no planejamento dos treinamentos de velocidade, a fim de que os atletas atinjam sua condição ótima nessa capacidade, melhorando o desempenho destes em ações de sprints em alta intensidade na ocasião do jogo. Desse modo, dividi-los em subgrupos nos quais os atletas apresentem características semelhantes, utilizando o método de análise pelo desempenho, 
é uma sugestão para direcionar o treinamento às necessidades destes atletas. Entretanto, se o intuito é potencializar o desempenho dos atletas de acordo com as exigências de jogo, o método de análise que separa os atletas por suas posições pode ser mais interessante para alcançar os objetivos propostos, pois sabendo-se das exigências de cada posição, o foco do treinamento poderá ser diferenciado entre elas.

A utilização de cronômetro manual para obtenção dos tempos no teste de velocidade não é recomendada quando utilizado estes métodos, devido à grande probabilidade de erros. Neste caso, o uso de células fotoelétricas para medidas de velocidade é importante para minimizar os erros de mensuração no teste. Esta parece constituir a principal limitação dos métodos apresentados, visto que este equipamento é compreendido como sendo de custo elevado para a realidade da maioria das equipes do futebol brasileiro. A literatura indica, no entanto, que quando não for possível o uso de equipamentos mais precisos para medidas de velocidade, a utilização de dois ou mais testes lineares máximos, em diferentes distâncias, são uma boa opção, sendo as distâncias de 10-40m, representativas para jogadores de futebol ${ }^{16,17,30}$.

Sugere-se, assim, a aplicação prática dos métodos abordados neste estudo e que outros estudos devam ser realizados, com amostras de tamanhos variados e com atletas de diferentes níveis competitivos, visando esclarecer os aspectos referentes aos dois métodos utilizados, no presente estudo, para análise da velocidade linear em jogadores de futebol.

\section{CONCLUSÃO}

Conforme os objetivos estabelecidos para o presente estudo, podemos inferir que, o método que separa os subgrupos pelo desempenho no teste linear de velocidade discrimina melhor as diferenças entre cada atleta ou subgrupo e o método que separa os subgrupos por suas posições no jogo discrimina melhor a característica de cada posição.

A utilização, em conjunto, desses dois métodos de análise da velocidade linear de jogadores de futebol pode potencializar a prescrição de treinamento, otimizando o desempenho de todos os atletas da equipe. É possível, também, identificar o foco de treinamento de velocidade para cada subgrupo e individualmente, ou de acordo com suas funções no jogo.

\section{REFERÊNCIAS BIBLIOGRÁFICAS}

1. Stølen T, Chamari K, Castagna C, Wisløff U. Physiology of Soccer: An Update. Sports Med 2005;35(6):501-536.
2. Rampinini E, Bishop D, Marcora SM, Bravo DF, Sassi R, Impellizzeri FM. Validity os Simple Field Tests as Indicators as Match-Related Physical Performance in Top-Level Professional Soccer Players. Int J Sports Med 2007;28(3):228-235.

3. Mohr M, Krustrup P, Bangsbo J. Match Performance of High-Standard Soccer Players With Special Reference to Development of Fatigue. J Sports Sci 2003;21(7):519-528.

4. Di Salvo V, Baron R, Tschan H, Montero JFC, Bachl N, Pigozzi F. Performance Characteristics According to Playing Position in Elite Soccer. Int J Sports Med 2007;28(3):222-227.

5. Barros RML, Misuta MS, Menezes RP, Fiqueroa PJ, Moura FA, Cunha SA, et al. Analysis of the Distances Covered by First Division Brazilian Soccer Players Obtained with an Automatic Tracking Method. J Sports Sci Med 2007;6(2):233-242.

6. Reilly T, Bangsbo J, Franks A. Anthropometric and Physiological Predispositions for Elite Soccer. J Sports Sci 2000;18(9):669-683.

7. Bloomfield J, Polman R, O'Donoghue P. Physical Demands of Different Positions in FA Premier League Soccer. J Sports Sci Med 2007;6(1):63-70.

8. Bangsbo J. The Physiology of Soccer: With Special Reference to Intense Intermittent Exercise. Acta Physiol Scand 1994;15(Suppl. 619):1-156.

9. Svensson M, Drust B. Testing Soccer Players. J Sports Sci 2005;23(6):601-618.

10. Little T, Williams A. Specificity of Acceleration, Maximum Speed, and Agility in Professional Soccer Players. J Strength Cond Res 2005;19(1):76-78.

11. Murphy AJ, Lockie RG, Coutts AJ. Kinematic Determinants of Early Acceleration in Field Sport Athletes. J Sport Sci Med 2003;2(4):144-150.

12. Vázquez AV, Gayo AA. Aproximación Conceptual a la Velocidad en Deportes de Equipo: El Caso Fútbol. Apunts 2002;69(3):44-58.

13. Reilly T. Energetics of High-Intensity Exercise (Soccer) With Particular Reference to Fatigue. J Sports Sci 1997;15(3):257-263.

14. Bangsbo J, Nørregaard L, Thors $\varnothing$ F. Activity Profile of Competition Soccer. Can J Sport Sci 1991;16(2):110116.

15. Valquer W, Barros TL, Sant'anna M. High Intensity Motion Pattern Analyses of Brazilian Elite Soccer Players (Abstract). IV Congress of National Analysis of Sport. Lisbon:1998, p. 80.

16. Wisløff U, Castagna C, Helgerud J, Jones R, Hoff J. Strong Correlation of Maximal Squat Strength with Sprint Performance and Vertical Jump Height in Elite Soccer Players. Br J Sports Med 2004;38(3):285-288.

17. Cometti G, Maffiuletti NA, Pousson M. Isokinetic Strength and Anaerobic Power of Elite, Subelite and Amateur French Soccer Players. Int J Sports Med 2001;22(1):45-51.

18. Brown TD, Vescovi JD, VanHeest JL. Assesment of Linear Sprinting Performance: Theoretical Paradigm. J Sports Sci Med 2004;3(4):203-210. 
19. Cissik JM. Means and Methods of Speed Training, Part II. National Strength Cond Assoc 2005;27(1):18-25.

20. Di Salvo V, Pigozzi F. Physical Training of Football Players Based on Their Positional Rules in The Team. J Sports Med Phys Fitness 1998;38(4):294-297.

21. Dauty M, Bryand F, Josse MP. Relation Entre la Force Isocinétique, le Saut et le Sprint Chez le Footballeur de Haut Niveau. Sci Sports 2002;17(3):122-127.

22. Dourado AC, Stanganelli LCR, Daros LB, Frisseli A, Montanholi AF, Osieck R. Assessment of Anthropometric Characteristics and Sprint Velocity in Soccer Players From 5 Different Age Groups. J Sport Sci Med 2007;6(Suppl. 10):136-137.

23. Neves AP, Barros J, Ribeiro VB. Correlation Between Strength and Power and Short Sprint Performance on Soccer Players. Med Sci Sports Exerc 1999;31(Suppl.):78.

24. Newman MA, Tarpenning KM, Marino FE. Relashionships Between Isokinetic Knee Strength, Single-Sprint Performance, and Repeated-Sprint Ability in Football Players. J Strength Cond Res 2004;18(4):867-872.

25. Strudwick A, Reilly T, Doran D. Anthropometric and Fitness Profiles of Elite Players in Two Football Codes. J Sports Med Phys Fitness 2002;42(2):239-242.

26. Neto LGS, Nunes CG, Hespanhol JE, Arruda M. Change in Fitness Variables of Professional Brazilian
Soccer Players in Preseason. J Sport Sci Med 2007;6(Suppl. 10):170-171.

27. Rábago JMC, Vicente JGV, Pascual CM, Garcia-López J. Influencia del entrenamiento de pretemporada en la fuerza explosiva y velocidad de un equipo profesional y otro amateur de un mismo club de fútbol. Apunts 2001(63):46-52.

28. Neto L, Nunes C, Hespanhol J, Arruda M. Physiological and Anthropometric Characteristic of Junior Brazilian Soccer Players (Abstract). World Congress on Science and Football - 5. Lisbon: 2003, p. 318.

29. Pasquarelli BN, Stanganelli LCR, Dourado AC, Teixeira SHE, Duarte ACV. Análise da Velocidade Linear de 10 metros em Jogadores de Futebol (Resumo). XXX Simpósio Internacional de Ciências do Esporte. São Paulo: 2007, p. 269.

30. Hoff J. Training and Testing Physical Capacities for Elite Soccer Players. J Sport Sci 2005;23(6):573-582.

\section{Endereço para correspondência}

Bruno Natale Pasquarelli

Avenida Rui Barbosa, 2412, Santana

12212-000 - São José dos Campos, SP. Brasil

E-mail: brunopasquarelli@hotmail.com 